\section{The Univariate Closure Conditions of All Fully-Parallel Planar Robots Derived From a Single Polynomial}

\author{
Nicolás Rojas and Federico Thomas, Member, IEEE
}

\begin{abstract}
The real roots of the univariate polynomial closure condition of a planar parallel robot determine the solutions of its forward kinematics. This paper shows how the univariate polynomials of all fullyparallel planar robots can be derived directly from that of the widely known 3-RPR robot by simply formulating these polynomials in terms of distances and oriented areas. This is a relevant result because it avoids the case-by-case treatment that requires different sets of variable eliminations to obtain the univariate polynomial of each fully-parallel planar robot.
\end{abstract}

Index Terms-Planar robots, forward kinematics, position analysis, planar Gough-Stewart platforms.

\section{INTRODUCTION}

A fully-parallel planar robot consists of a moving platform connected to a fixed base by three serial kinematic chains, or legs. Each chain consists of three independent 1-degree-of-freedom lower pair joints, one of which is driven by an actuator. Since the displacement of the moving platform is confined to a plane, only revolute $(\mathrm{R})$ and prismatic $(\mathrm{P})$ pairs are considered. Then, the topology of each serial kinematic chain can be described by three letters. There are seven possible combinations: RRR, RPR, RRP, PRR, RPP, PRP, and PPR. The chain PPP is not considered because three $P$ pairs represent three translations in the plane which cannot be independent. The actuated joint is identified by underlining it. Then, since any of the three joints can be actuated, there are twenty one possible legs which can be grouped in four leg architectures — known as the RR, PR, RP and PP legs (see Table \- attending to the sequence of passive joints. The number of passive prismatic joints in a loop cannot be more than three, otherwise the robot would gain one degree of freedom. This fact limits the number of possible PP legs to one. Then, since there are $\left(\begin{array}{c}18 \\ 3\end{array}\right)=1140$ feasible combinations of legs of type RR, PR, and RP, and $3 \cdot\left(\begin{array}{c}18 \\ 2\end{array}\right)=513$ feasible combinations of one leg of type PP with any two legs of the other three types, the total number of different planar fully-parallel robots is 1653 . When the three actuators are locked, the robot becomes a rigid structure provided that it is not in a singularity. This permits to classify these 1653 robots into 10 classes attending to the topology of the resulting structure (see Table II). Up to this point, we have followed the usual approach used in robotics to present all possible fully-parallel planar robots but observe that the resulting structures are nothing more than the 5-link Assur kinematic chains, also known as the planar Assur II groups [1].

The forward kinematics of parallel robots consists in finding the possible poses of the mobile platform, for specified values of the actuated joint coordinates, with respect to the fixed base. Thus, it reduces to the position analysis of one of the ten possible structures in Table 1 Numerical solutions to this problem are enough for many applications but yield little insight into the problem. The alternative are the exact methods which rely on the computation of a univariate

The authors are with the Institut de Robòtica i Informàtica Industrial (CSIC-UPC), Llorens Artigas 4-6, 08028 Barcelona, Spain. E-mails: \{nrojas, fthomas\}@iri.upc.edu.

This paper has supplementary downloadable multimedia material. This material consists of a Maple Worksheet containing the example presented in Section IV No particular requirements, except for an installed copy of Maple, version 12.0 or higher. Contact the first author for further questions about this material.

This work was supported by the Spanish Ministry of Economy and Competitiveness through the Explora programme under Contract DPI201113208-E.
TABLE I

PLANAR FULLY-PARALLEL ROBOT LEG TYPES

\begin{tabular}{|c|c|c|c|}
\hline Type RR & Type RP & Type PR & Type PP \\
\hline RRR & RPR & RPR & \multirow{6}{*}{$\begin{array}{l}\underline{R P P} \\
\text { PRP } \\
\text { PPR }\end{array}$} \\
\hline $\bar{R} \underline{R} R$ & $\mathrm{R} \underline{\bar{P}} \overline{\mathrm{P}}$ & $\overline{\mathrm{P}} \underline{R} R$ & \\
\hline $\mathrm{R} \bar{R} \underline{R}$ & $\mathrm{R} \bar{P} \underline{P}$ & PR $\underline{R}$ & \\
\hline $\mathrm{R} \underline{\mathrm{P}} \overline{\mathrm{R}}$ & $\mathrm{PR} \overline{\mathrm{P}}$ & $\mathrm{PR} \overline{\mathrm{P}}$ & \\
\hline$\underline{\mathrm{P}} \overline{\mathrm{R}} \mathrm{R}$ & $\underline{\bar{R}} R P$ & $\underline{P P} \bar{R}$ & \\
\hline RRP & $\bar{R} \underline{R} P$ & $\overline{\mathrm{P}} \underline{\mathrm{P} R}$ & \\
\hline
\end{tabular}

polynomial thus providing what it is usually called a closed-form solution to the problem.

In 1987, $\mathrm{Li}$ and Matthew solved the position analysis problem of the ten 5-link Assur kinematic chains in closed form for the first time [2]. Their approach consisted in realizing that every Assur II group consisted of two kinematically independent loops which can be classified into only six types: RRRR, RPRR, RPPR, RPRP, RRPP, and RPPP. Then, they reduced the problem to obtain the loop equations for these six loops in general form and compute the resultant in a single variable for the ten feasible combinations. Although outstanding in many ways for its time (the authors even envisaged the possibility of applying their results to planar and spherical robots), this work has been overlooked by the robotics community. In 1996, Merlet tackled the same problem from a different point of view which resulted in a case-by-case analysis [3]. Other solutions for some 5-link Assur kinematic chains have been presented, at least, in [4], [5], [6], [7]. The development of a remarkable unified formulation for the forward kinematics of all fully-parallel planar robots started in 1995 with Husty's first use of the Grünwald-Blaschke kinematic mapping to solve the forward kinematics of the 3-RPR robot [8]. This formulation was thereafter extended by Hayes, Chen, Zsombor-Murray, and Husty himself who presented their results in a series of publications that culminated with a recent monograph [9], [10], [11], [12], [13], [14], [15], [16], [17]. The approach followed by these authors is based on examining the motion of each leg separately which can be represented by only three types of surfaces: an hyperbolic paraboloid (for legs of type PR and RP), a hyperboloid of one sheet (for legs of type $\mathrm{RR}$ ), or a plane (for legs of type PP). Then, the forward kinematics problem boils down to find the points of intersection of these three such surfaces. The result is indeed a uniform procedure for solving the forward kinematics of all fully-parallel planar robots but an elimination process is still required to obtain a univariate polynomial for 11 different cases.

A different unifying approach stems from regarding a translational motion as an infinitely small rotation about a point at infinity. It is well-known that a translation in the direction $\left(u_{x}, u_{y}\right)$ may be represented as a rotation about the ideal point given in homogeneous coordinates by $\left(-u_{y}, u_{x}, 0\right)$. This is probably the most simple unifying approach to deal with revolute and prismatic joints simultaneously but, using the standard formulations such as those based on independent loop equations, it is difficult to be accommodated. This paper is essentially devoted to show how a coordinate-free formulation based on distances and oriented areas provides a framework within which this idea can be easily applied. This will allow us to conclude that the univariate polynomial of the 3-RPR robot contains all the necessary and sufficient information for solving the forward kinematics of all fully-parallel planar robots.

This paper is organized as follows. Section $\Pi$ briefly reviews the basics of deriving the univariate polynomial closure condition of the 3-RPR robot in terms of distances and oriented areas. Section III shows how to transform any other fully-parallel robot with prismatic joints into a 3-RPR robot with some revolute joint centers located 
TABLE II

THE 10 FULLY-PARALLEL PLANAR ROBOT FAMILIES

\begin{tabular}{|c|c|c|}
\hline $\begin{array}{l}\text { Robot family } \\
\text { (\# of different } \\
\text { robot topologies) }\end{array}$ & $\begin{array}{l}\text { Associated } \\
\text { structure }\end{array}$ & Leg types \\
\hline I (56) & & RR-RR-RR \\
\hline II (252) & & $\begin{array}{l}\text { RP-RR-RR } \\
\text { PR-RR-RR }\end{array}$ \\
\hline III (216) & & RP-PR-RR \\
\hline IV (252) & & $\begin{array}{l}\text { RP-RP-RR } \\
\text { PR-PR-RR }\end{array}$ \\
\hline V (252) & & $\begin{array}{l}\text { RP-RP-PR } \\
\text { PR-PR-RP }\end{array}$ \\
\hline VI (63) & & PP-RR-RR \\
\hline VII (216) & & $\begin{array}{l}\text { PP-RR-RP } \\
\text { PP-RR-PR }\end{array}$ \\
\hline VIII (112) & & $\begin{array}{l}\text { RP-RP-RP } \\
\text { PR-PR-PR }\end{array}$ \\
\hline IX (108) & & PP-PR-RP \\
\hline X (126) & & $\begin{array}{l}\text { PP-RP-RP } \\
\text { PP_PR_PR }\end{array}$ \\
\hline
\end{tabular}

at infinity. Section IV shows through examples how to solve using the univariate polynomial of the 3-RPR robot- the position analysis of different fully-parallel planar robots. Finally, we conclude in Section $\nabla$ with a summary of the main contributions and prospects for future research.

II. THE UNIVARIATE POLYNOMIAL CLOSURE CONDITION OF THE 3-RPR ROBOT IN TERMS OF DISTANCES AND ORIENTED AREAS

In what follows, $P_{i}$ will denote a point, $\overline{P_{i} P_{j}}$ the segment defined by $P_{i}$ and $P_{j}$, and $\triangle P_{i} P_{j} P_{k}$ the triangle defined by $P_{i}, P_{j}$, and $P_{k}$.
Moreover, $\mathbf{p}_{i j}=\overrightarrow{P_{i} P_{j}}$ and $s_{i, j}=d_{i, j}^{2}=\left\|\mathbf{p}_{i, j}\right\|^{2}$.

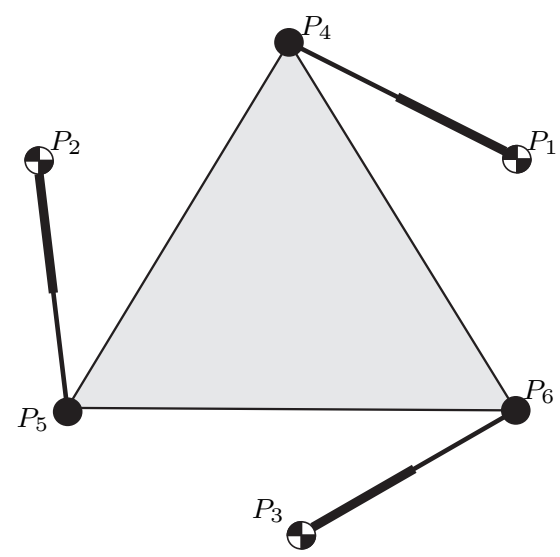

Fig. 1. The general 3-RPR planar robot platform.

Figure 1 shows a general 3-RPR planar robot platform. The center of the three grounded passive revolute joints, $\rightarrow$, define the base oriented triangle $\triangle P_{1} P_{3} P_{2}$ and the three moving passive revolute joints centers, $\bullet$, the moving oriented triangle $\triangle P_{6} P_{4} P_{5}$. The squared lengths of the active prismatic joints are $s_{1,4}, s_{2,5}$, and $s_{3,6}$. Much has been written about this parallel robot because of its practical interest, mechanical simplicity, and rich mathematical properties. A review of the different techniques for solving the forward kinematics of this robot can be found in [18] where it is shown that this problem can be solved by finding the roots of:

$$
s_{2,5}=\operatorname{det}\left(\mathbf{I}-\mathbf{Z}_{1,3,2} \mathbf{Z}_{1,6,3}-\mathbf{Z}_{6,4,5} \mathbf{Z}_{6,1,4}\right) s_{1,6}
$$

where

$$
\mathbf{Z}_{i, j, k}=\frac{1}{2 s_{i, j}}\left[\begin{array}{cc}
s_{i, j}+s_{i, k}-s_{j, k} & -4 A_{i, j, k} \\
4 A_{i, j, k} & s_{i, j}+s_{i, k}-s_{j, k}
\end{array}\right]
$$

with

$$
A_{i, j, k}= \pm \frac{1}{4} \sqrt{\left(s_{i, j}+s_{i, k}+s_{j, k}\right)^{2}-2\left(s_{i, j}^{2}+s_{i, k}^{2}+s_{j, k}^{2}\right)}
$$

being the oriented area of $\triangle P_{i} P_{j} P_{k}$ (defined as positive if $P_{i}, P_{j}$, and $P_{k}$ are ordered counter-clockwise, and negative otherwise).

Equation (1) expresses the set of values of $s_{1,6}$ compatible with the fixed lengths of the active joints, the base and moving platform side lengths, and the signs of the oriented triangles $\triangle P_{1} P_{3} P_{2}$ and $\triangle P_{6} P_{4} P_{5}$. The expansion of the determinant in this equation yields a scalar radical equation as a function of the unknown squared distance $s_{1,6}$ which, after clearing radicals, finally yields the univariate polynomial closure condition of the robot, a 6th-degree polynomial equation in $s_{1,6}$ which will be denoted by $\Gamma\left(s_{1,6}\right)=0$. The real roots of this polynomial determine the solutions of the forward kinematics. The interested reader is referred to $[18]$ for details on the above derivation.

\section{REPLACING REVOLUTE BY PRISMATIC JOINTS}

In this section, we will consider the case in which the three revolute joints connected to the moving platform are replaced with prismatic joints. We will proceed progressively by replacing first one, then two, and finally the three revolute joints. At the end, it will become clear that all other cases can be easily deduced from this analysis. 

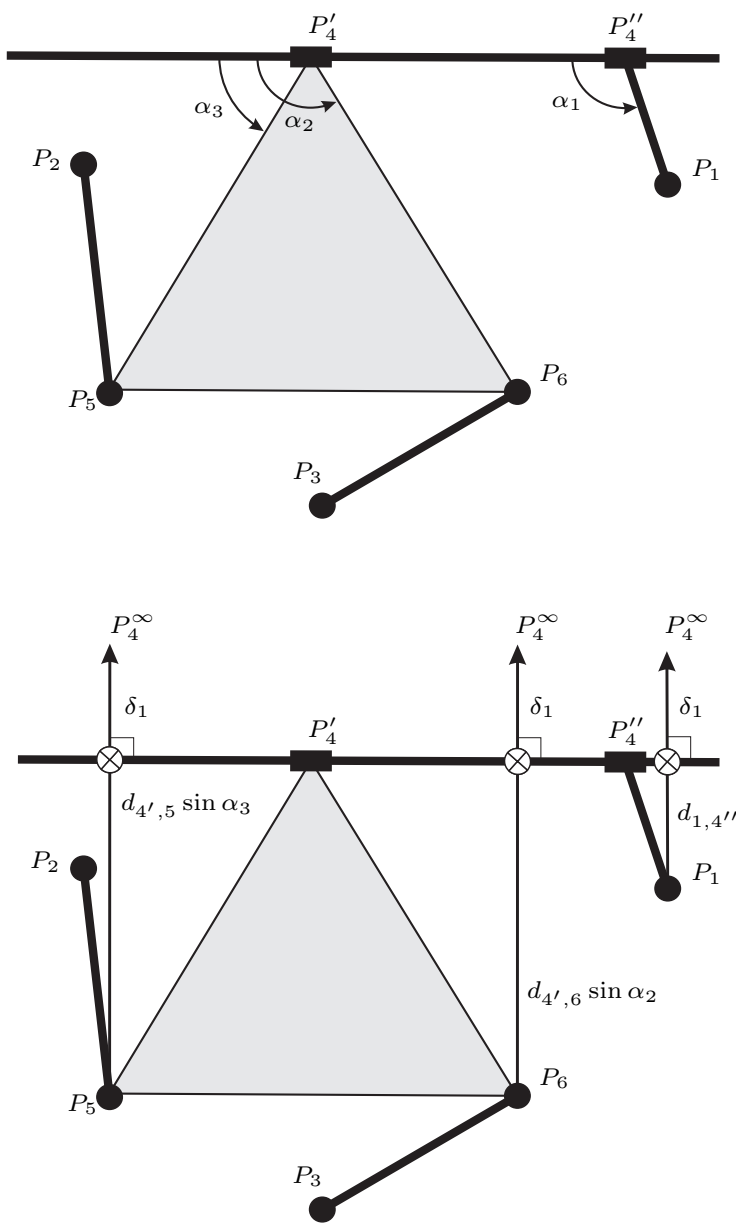

Fig. 2. One revolute joint in the moving platform is substituted by a prismatic joint.

\section{A. Replacing one revolute joint}

Let us suppose that the revolute joint centered at $P_{4}$ in Fig. 1 is replaced with a prismatic joint, as shown in Fig. 2 top), such that $P_{4}$ is split into $P_{4}^{\prime}$ and $P_{4}^{\prime \prime}$. This new joint is placed at fixed orientations with respect to the links connected to them. Once an orientation is assigned to the prismatic joint axis with respect to its adjacent links, a set of orientation angles can be defined (in this case $\alpha_{1}, \alpha_{2}$ and $\alpha_{3}$ ) and, as a consequence, an oriented distance can be defined between $P_{1}, P_{5}$ and $P_{6}$ and the prismatic joint axis. This defines a set of new points on this axis: those that realize the minimum distance to $P_{1}, P_{5}$ and $P_{6}$ which are denoted by $\otimes$ in Fig. 2 bottom). Note that the prismatic joint imposes the alignment of these points but, for the moment, let us suppose that they all are located at the same distance, say $\delta_{i}$, from $P_{4}^{\infty}$. This would imply that they would lie on a circle but, if $\delta_{i} \rightarrow \infty$, they would again lie on a line as imposed by the prismatic join. Actually, the presented geometric transformation simply consists in replacing $P_{4}$ by $P_{4}^{\infty}$ and substituting the distances between $P_{4}$ and $P_{1}, P_{5}$, and $P_{6}$, by

$$
\begin{aligned}
& d_{4,1}=\delta_{1}+d_{1,4^{\prime \prime}} \sin \alpha_{1}, \\
& d_{4,5}=\delta_{1}+d_{4^{\prime}, 5} \sin \alpha_{3}, \\
& d_{4,6}=\delta_{1}+d_{4^{\prime}, 6} \sin \alpha_{2},
\end{aligned}
$$

respectively.

It is worth noting that, after the described geometric transformation, it might happen that the orientation of $\triangle P_{4}^{\infty} P_{5} P_{6}$ have changed with respect to that of $\triangle P_{4} P_{5} P_{6}$. This has to be taken into account in the univariate polynomial by changing the sign of $A_{6,4,5}$ if needed.

After the introduced changes, the univariate polynomial closure condition can be rewritten as a polynomial in $\delta_{1}$, that is,

$$
\Gamma\left(s_{1,6}\right) \mid \begin{aligned}
& s_{1,4}=\left(\delta_{1}+d_{1,4^{\prime \prime}} \sin \alpha_{1}\right)^{2} \\
& s_{4,5}=\left(\delta_{1}+d_{4^{\prime}, 5} \sin \alpha_{3}\right)^{2} \\
& s_{4,6}=\left(\delta_{1}+d_{4^{\prime}, 6} \sin \alpha_{2}\right)^{2}
\end{aligned}=\sum_{i=0}^{n} \gamma_{i}\left(s_{1,6}\right) \delta_{1}^{i}=0 .
$$

Now, factoring out $\delta_{1}^{n}$ in the above closure condition, we get

$$
\delta_{1}^{n}\left(\gamma_{n}\left(s_{1,6}\right)+\frac{\gamma_{n-1}\left(s_{1,6}\right)}{\delta_{1}}+\frac{\gamma_{n-2}\left(s_{1,6}\right)}{\delta_{1}^{2}}+\ldots\right)=0
$$

Then, the closure condition can be expressed as:

$$
\gamma_{n}\left(s_{1,6}\right)+\frac{\gamma_{n-1}\left(s_{1,6}\right)}{\delta_{1}}+\frac{\gamma_{n-2}\left(s_{1,6}\right)}{\delta_{1}^{2}}+\cdots=0 .
$$

Since $\delta_{1}$ is finally made to tend to infinity, we conclude that the univariate polynomial closure condition for the resulting robot, after the introduction of a prismatic joint, is $\gamma_{n}\left(s_{1,6}\right)=0$.

\section{B. Replacing two revolute joints}

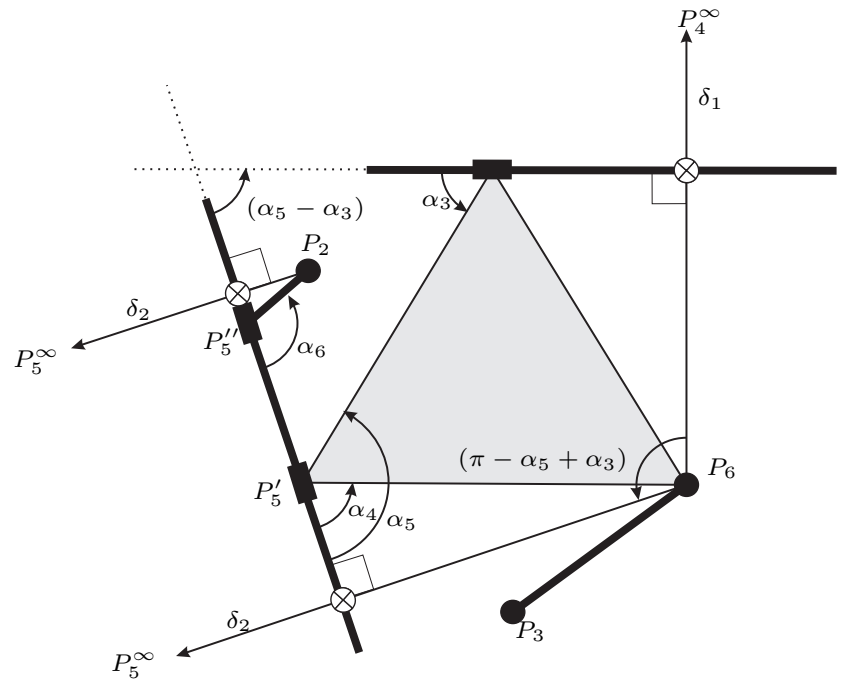

Fig. 3. Two revolute joints connected to the moving platform are substituted by prismatic joints.

Now, let us suppose that the revolute joint centered at $P_{5}$ is also replaced with a prismatic joint as indicated in Fig. 3 . Following the same reasoning used above, this is equivalent to replacing $P_{5}$ with a point at infinity, $P_{5}^{\infty}$, and substituting the distances between $P_{5}$ and $P_{2}$, and $P_{6}$, by

$$
\begin{aligned}
& d_{5,2}=\delta_{2}+d_{5^{\prime \prime}, 2} \sin \alpha_{6}, \\
& d_{5,6}=\delta_{2}+d_{5^{\prime}, 6} \sin \alpha_{4},
\end{aligned}
$$

with $\delta_{2} \rightarrow \infty$, respectively. To obtain the substitution for $d_{4,5}$, observe that the angle formed by the two prismatic joints is $\alpha_{5}-\alpha_{3}$. Then,

$$
\angle P_{5}^{\infty} P_{6} P_{4}^{\infty}=\pi-\alpha_{5}+\alpha_{3} .
$$

Therefore, using the law of cosines, we conclude that:

$$
\begin{aligned}
d_{4,5}^{2}= & d_{5,6}^{2}+d_{4,6}^{2}-2 d_{5,6} d_{4,6} \cos \left(\pi-\alpha_{5}+\alpha_{3}\right) \\
= & \left(\delta_{2}+d_{5^{\prime}, 6} \sin \alpha_{4}\right)^{2}+\left(\delta_{1}+d_{4^{\prime}, 6} \sin \alpha_{2}\right)^{2} \\
& +2\left(\delta_{2}+d_{5^{\prime}, 6} \sin \alpha_{4}\right)\left(\delta_{1}+d_{4^{\prime}, 6} \sin \alpha_{2}\right) \cos \left(\alpha_{5}-\alpha_{3}\right) .
\end{aligned}
$$


with $\delta_{1} \rightarrow \infty$ and $\delta_{2} \rightarrow \infty$.

\section{Replacing three revolute joints}

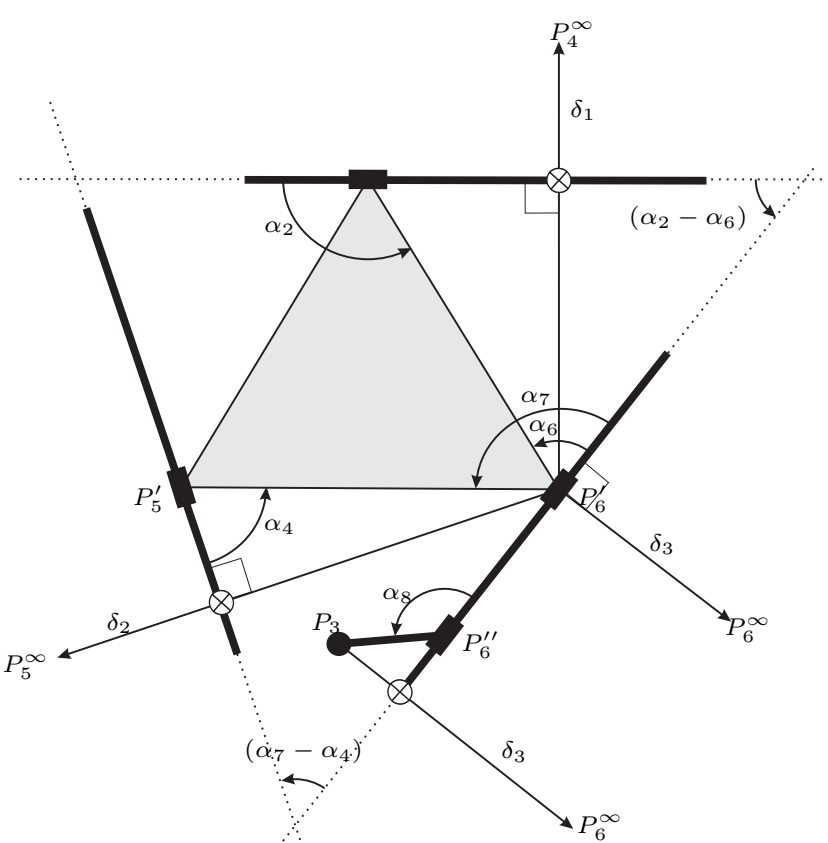

Fig. 4. The three revolute joints connected to the moving platform are substituted by prismatic joints.

Finally, let us assume that the revolute joint centered at $P_{6}$ is also replaced with a prismatic joint with the orientation angles indicated in Fig. 4 Using the same reasoning as above, this is equivalent to take $P_{6}$ to infinity and substitute the distance between $P_{6}$ and $P_{3}$ by

$$
d_{3,6}=\delta_{3}+d_{3,6^{\prime \prime}} \sin \alpha_{8} .
$$

In this case, to obtain the substitutions for the distances between $P_{6}$ and $P_{5}$, and $P_{4}$, observe that the angles formed by the new prismatic joint axis with those defined by the other two are $\alpha_{7}-\alpha_{4}$ and $\alpha_{2}-\alpha_{6}$ (see Fig. 4). Then,

$$
\begin{aligned}
& \angle P_{5}^{\infty} P_{6}^{\prime} P_{6}^{\infty}=\pi-\alpha_{7}+\alpha_{4}, \\
& \angle P_{6}^{\infty} P_{6}^{\prime} P_{4}^{\infty}=\pi-\alpha_{2}+\alpha_{6} .
\end{aligned}
$$

Consequently, using the law of cosines,

$$
\begin{aligned}
d_{5,6}^{2}= & \left(\delta_{2}+d_{5^{\prime}, 6^{\prime}} \sin \alpha_{4}\right)^{2}+\delta_{3}^{2} \\
& +2\left(\delta_{2}+d_{5^{\prime}, 6^{\prime}} \sin \alpha_{4}\right) \delta_{3} \cos \left(\alpha_{7}-\alpha_{4}\right), \\
d_{6,4}^{2}= & \left(\delta_{1}+d_{4^{\prime}, 6^{\prime}} \sin \alpha_{2}\right)^{2}+\delta_{3}^{2} \\
& +2\left(\delta_{1}+d_{4^{\prime}, 6^{\prime}} \sin \alpha_{2}\right) \delta_{3} \cos \left(\alpha_{2}-\alpha_{6}\right),
\end{aligned}
$$

and

$$
d_{4,5}^{2}=d_{5,6}^{2}+d_{4,6}^{2}+2 d_{5,6} d_{4,6} \cos \left(\alpha_{5}+\alpha_{3}\right) .
$$

The required substitutions for all other cases can be derived following the same procedure. Table III compiles them all thus concluding that the univariate polynomials of all fully-parallel planar robots can be deduced from the univariate polynomial of the 3-RPR robot when expressed in terms of distances and oriented areas. The degree of the resulting univariate polynomial for each family, that is, the maximum number of solutions of the forward kinematics, is also included in Table III and denoted by FK. The practical consequences of the presented formulation are better understood through an example.

\section{EXAMPLE}

Let us consider the planar 3-RPR parallel robot in Fig. 1 As an example, let us set $s_{1,2}=16, s_{1,3}=65, s_{2,3}=73, s_{5,6}=25$, $s_{4,6}=25, s_{4,5}=36$, and squared input joints $s_{1,4}=1, s_{2,5}=121$, and $s_{3,6}=169$. Let us also assume that the orientation of $\triangle P_{1} P_{2} P_{3}$ and $\triangle P_{6} P_{4} P_{5}$ are positive. Substituting these values in $\Gamma\left(s_{1,6}\right)$, the following univariate polynomial is obtained

$$
\begin{aligned}
& s_{1,6}^{6}-293.1486 s_{1,6}^{5}+54084.9111 s_{1,6}^{4}-3.558710^{6} s_{1,6}^{3} \\
& +1.000410^{8} s_{1,6}^{2}-1.224010^{9} s_{1,6}+5.384310^{9} .
\end{aligned}
$$

This polynomial has a double real root at 32.0000 . The corresponding solution of the forward kinematics, for the case in which $P_{1}=(0,0)^{T}, P_{2}=(4,0)^{T}$, and $P_{3}=(1,8)^{T}$, appears in the first row of Fig. 5. This example was studied in [18] to compare the different formulations for solving the forward kinematic of the 3-RPR parallel robot presented in [19], [20], and [8], with that based on distances and oriented areas.

When the revolute joint centered at $P_{4}$ is replaced with a prismatic joint, a RPP-RPR-RPR planar robot is obtained. This kind of robot belongs to the type II robot family in Table IIII If the orientation of the passive prismatic joint with respect to its adjacent links, according to the notation used in Table III is given by

$$
\alpha_{1}=\frac{3}{4} \pi, \alpha_{2}=\frac{5}{4} \pi+\arctan \frac{4}{3}, \text { and } \alpha_{3}=\frac{5}{4} \pi,
$$

then

$$
d_{1,4}=\delta_{1}+\frac{1}{\sqrt{2}}, d_{4,5}=\delta_{1}-3 \sqrt{2}, \text { and } d_{4,6}=\delta_{1}-\frac{7}{\sqrt{2}} .
$$

Substituting these distances in $\Gamma\left(s_{1,6}\right)$, while keeping all other distances unaltered, and computing the leading coefficient of the resulting polynomial in $\delta_{1}$, we get the univariate polynomial

$$
\begin{aligned}
& s_{1,6}^{6}-672.0638 s_{1,6}^{5}+1.498310^{5} s_{1,6}^{4}-1.437210^{7} s_{1,6}^{3} \\
& +6.172910^{8} s_{1,6}^{2}-1.202310^{10} s_{1,6}+8.793410^{10} .
\end{aligned}
$$

The real roots of this polynomial are $32.0000,112.2332$, 141.1726, and 342.8691. The corresponding solutions of the forward kinematics appear in the second row of Fig. 5

Now, if the revolute joint centered at $P_{6}$ is also replaced with a prismatic joint, a RPP-RPP-RPR robot is obtained. This kind of robot belongs to the type IV robot family in Table III If this new prismatic joint orientation with respect to its adjacent links, according to the notation of Table III given by

$$
\alpha_{4}=2 \pi-\arctan \frac{4}{3}, \alpha_{5}=\pi+\arctan \frac{4}{3},
$$

and

$$
\alpha_{6}=\pi+\arctan \frac{12}{5}
$$

then

$$
\begin{aligned}
& d_{1,4}=\delta_{1}+\frac{1}{\sqrt{2}}, \\
& d_{4,5}=\delta_{1}-3 \sqrt{2}, \\
& d_{5,6}=\delta_{2}-4, \\
& d_{4,6}^{2}=\delta_{1}^{2}+\delta_{2}^{2}+\sqrt{2} \delta_{1} \delta_{2}-10 \sqrt{2} \delta_{1}-14 \delta_{2}+58, \\
& d_{3,6}=\delta_{2}-12, \\
& d_{1,6}=\delta_{2}-t .
\end{aligned}
$$

Substituting these values in $\Gamma\left(s_{1,6}\right)$, while keeping all other distances unchanged, and computing the leading coefficient of the resulting polynomial in $\delta_{1}$, and then the leading coefficient of the re- 
TABLE III

DISTANCE SUBSTITUTIONS FOR EACH ROBOT FAMILY

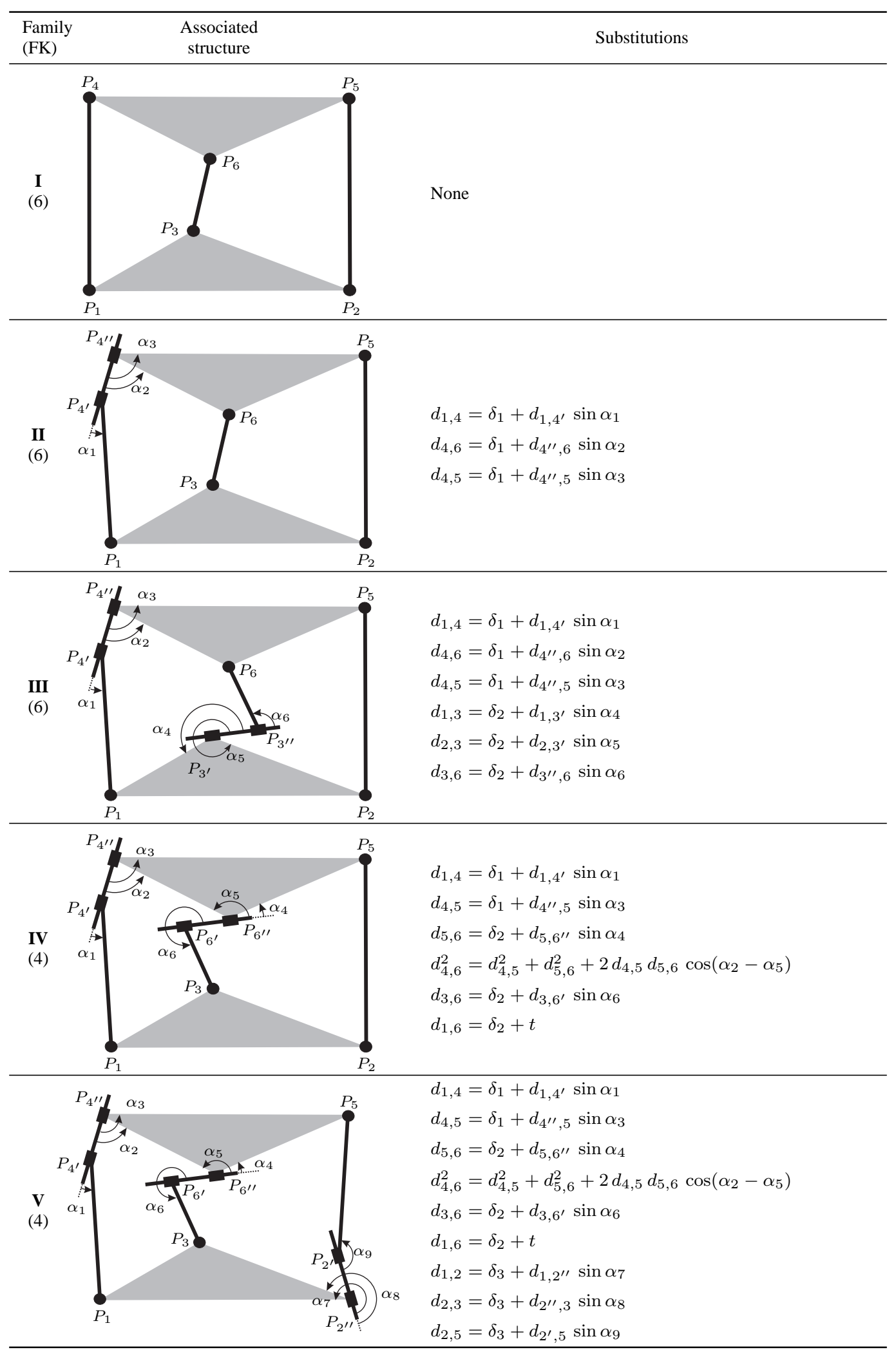


TABLE III (CONT'D)

DISTANCE SUBSTITUTIONS FOR EACH ROBOT FAMILY

\begin{tabular}{|c|c|c|}
\hline $\begin{array}{l}\text { Family } \\
\text { (FK) }\end{array}$ & $\begin{array}{l}\text { Associated } \\
\text { structure }\end{array}$ & Substitutions \\
\hline $\begin{array}{l}\text { VI } \\
(2)\end{array}$ & & $\begin{array}{l}d_{1,4}^{2}=\delta_{1}^{2}+\eta^{2}-2 \delta_{1} \eta \cos \left(\alpha_{6}-\alpha_{1}\right), \text { with } \eta=\delta_{2}+d_{1^{\prime \prime}, 4^{\prime}} \sin \alpha_{6} \\
d_{4,6}=\delta_{1}+d_{4^{\prime \prime}, 6} \sin \alpha_{2} \\
d_{4,5}=\delta_{1}+d_{4^{\prime \prime}, 5} \sin \alpha_{3} \\
d_{1,2}=\delta_{2}+d_{1^{\prime}, 2} \sin \alpha_{4} \\
d_{1,3}=\delta_{2}+d_{1^{\prime}, 3} \sin \alpha_{5} \\
d_{1,6}=\delta_{2}+t\end{array}$ \\
\hline $\begin{array}{l}\text { VII } \\
(2)\end{array}$ & & $\begin{array}{l}d_{1,4}^{2}=\delta_{1}^{2}+\eta^{2}-2 \delta_{1} \eta \cos \left(\alpha_{6}-\alpha_{1}\right), \text { with } \eta=\delta_{2}+d_{1^{\prime \prime}, 4^{\prime}} \sin \alpha_{6} \\
d_{4,6}=\delta_{1}+d_{4^{\prime \prime}, 6} \sin \alpha_{2} \\
d_{1,2}=\delta_{2}+d_{1^{\prime}, 2} \sin \alpha_{4} \\
d_{1,3}=\delta_{2}+d_{1^{\prime}, 3} \sin \alpha_{5} \\
d_{1,6}=\delta_{2}+t \\
d_{5,6}=\delta_{3}+d_{5^{\prime}, 6} \sin \alpha_{8} \\
d_{4,5}^{2}=d_{4,6}^{2}+d_{5,6}^{2}-2 d_{4,6} d_{5,6} \cos \left(\alpha_{7}-\alpha_{3}\right) \\
d_{2,5}=\delta_{3}+d_{2,5^{\prime \prime}} \sin \alpha_{9}\end{array}$ \\
\hline $\begin{array}{c}P_{4^{\prime}} \\
\text { VIII } \\
\text { (2) }\end{array}$ & & $\begin{array}{l}d_{1,4}=\delta_{1}+d_{1,4^{\prime}} \sin \alpha_{1} \\
d_{3,6}=\delta_{2}+d_{3,6^{\prime}} \sin \alpha_{6} \\
d_{1,6}=\delta_{2}+t \\
d_{4,5}^{2}=\delta_{3}^{2}+\eta_{1}^{2}-2 \delta_{3} \eta_{1} \cos \left(\alpha_{7}-\alpha_{3}\right), \text { with } \eta_{1}=\delta_{1}+d_{4^{\prime \prime}, 5^{\prime}} \sin \alpha_{3} \\
d_{5,6}^{2}=\delta_{3}^{2}+\eta_{2}^{2}-2 \delta_{3} \eta_{2} \cos \left(\alpha_{4}-\alpha_{8}\right), \text { with } \eta_{2}=\delta_{2}+d_{5^{\prime}, 6^{\prime \prime}} \sin \alpha_{4} \\
d_{4,6}^{2}=d_{4,5}^{2}+d_{5,6}^{2}+2 d_{4,5} d_{5,6} \cos \left(\alpha_{2}-\alpha_{5}\right) \\
d_{2,5}=\delta_{3}+d_{2,5^{\prime \prime}} \sin \alpha_{9}\end{array}$ \\
\hline & & $\begin{array}{l}d_{1,4}^{2}=\delta_{1}^{2}+\eta^{2}-2 \delta_{1} \eta \cos \left(\alpha_{6}-\alpha_{1}\right), \text { with } \eta=\delta_{2}+d_{1^{\prime \prime}, 4^{\prime}} \sin \alpha_{6} \\
d_{4,6}=\delta_{1}+d_{4^{\prime \prime}, 6} \sin \alpha_{2} \\
d_{1,2}=\delta_{2}+d_{1^{\prime}, 2} \sin \alpha_{4} \\
d_{1,6}=\delta_{2}+t \\
d_{5,6}=\delta_{3}+d_{5^{\prime}, 6} \sin \alpha_{8} \\
d_{4,5}^{2}=d_{4,6}^{2}+d_{5,6}^{2}-2 d_{4,6} d_{5,6} \cos \left(\alpha_{7}-\alpha_{3}\right) \\
d_{2,5}=\delta_{3}+d_{2,5^{\prime \prime}} \sin \alpha_{9} \\
d_{2,3}=\delta_{4}+d_{2,3^{\prime}} \sin \alpha_{11} \\
d_{1,3}^{2}=d_{1,2}^{2}+d_{2,3}^{2}-2 d_{1,2} d_{2,3} \cos \left(\alpha_{10}-\alpha_{5}\right) \\
d_{3,6}=\delta_{4}+d_{3^{\prime \prime}, 6} \sin \alpha_{12}\end{array}$ \\
\hline $\begin{array}{c}\mathbf{X} \\
(1)\end{array}$ & & $\begin{array}{l}d_{1,4}^{2}=\delta_{1}^{2}+\eta^{2}-2 \delta_{1} \eta \cos \left(\alpha_{6}-\alpha_{1}\right), \text { with } \eta=\delta_{2}+d_{1^{\prime \prime}, 4^{\prime}} \sin \alpha_{6} \\
d_{4,6}=\delta_{1}+d_{4^{\prime \prime}, 6} \sin \alpha_{2} \\
d_{4,5}=\delta_{1}+d_{4^{\prime \prime}, 5} \sin \alpha_{3} \\
d_{1,6}=\delta_{2}+t \\
d_{2,3}=\delta_{3}+d_{2,3^{\prime}} \sin \alpha_{8} \\
d_{3,6}=\delta_{3}+d_{3^{\prime \prime}, 6} \sin \alpha_{9} \\
d_{1,2}^{2}=\delta_{4}^{2}+\eta_{1}^{2}-2 \delta_{4} \eta_{1} \cos \left(\alpha_{10}-\alpha_{4}\right), \text { with } \eta_{1}=\delta_{2}+d_{1^{\prime}, 2^{\prime \prime}} \sin \alpha_{4} \\
d_{2,3}^{2}=\delta_{4}^{2}+\eta_{2}^{2}+2 \delta_{4} \eta_{2} \cos \left(\alpha_{11}-\alpha_{8}\right), \text { with } \eta_{2}=\delta_{3}+d_{2^{\prime \prime}, 3^{\prime}} \sin \alpha_{8} \\
d_{1,3}^{2}=d_{1,2}^{2}+d_{2,3}^{2}-2 d_{1,2} d_{2,3} \cos \left(\alpha_{7}-\alpha_{5}\right) \\
d_{2,5}=\delta_{4}+d_{2^{\prime}, 5} \sin \alpha_{12}\end{array}$ \\
\hline
\end{tabular}




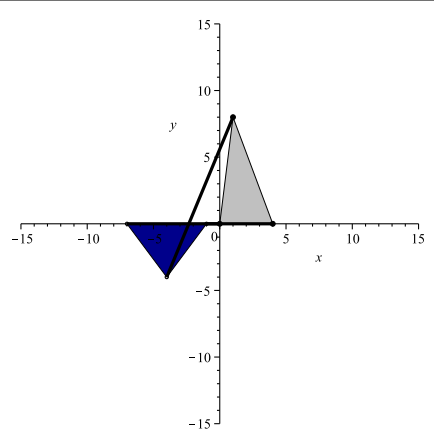

$s_{1,6}=32.0000$
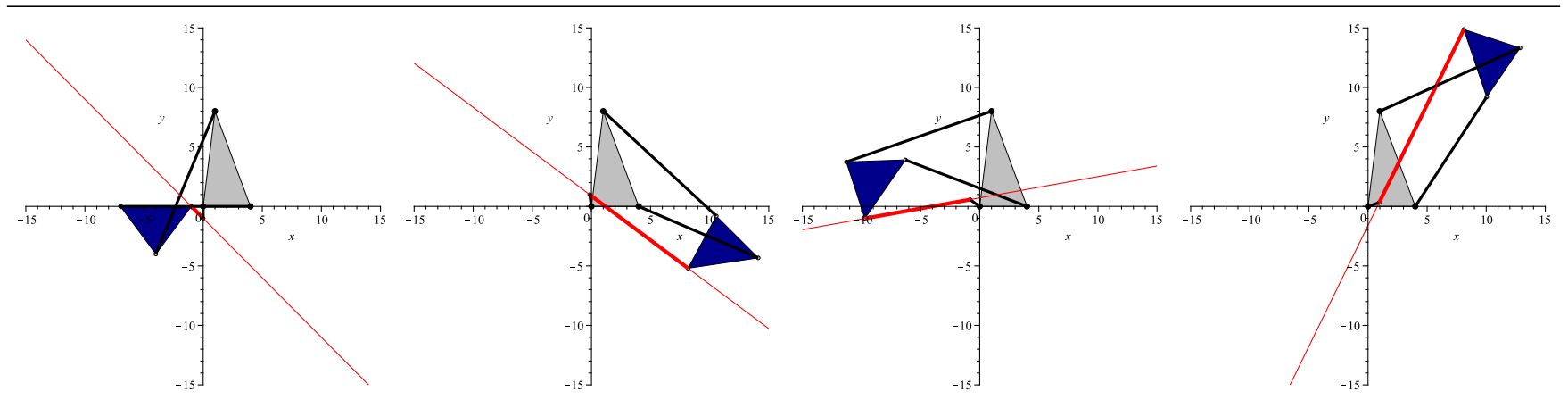

$s_{1,6}=32.0000$

$s_{1,6}=112.2332$

$s_{1,6}=141.1726$

$s_{1,6}=342.8691$
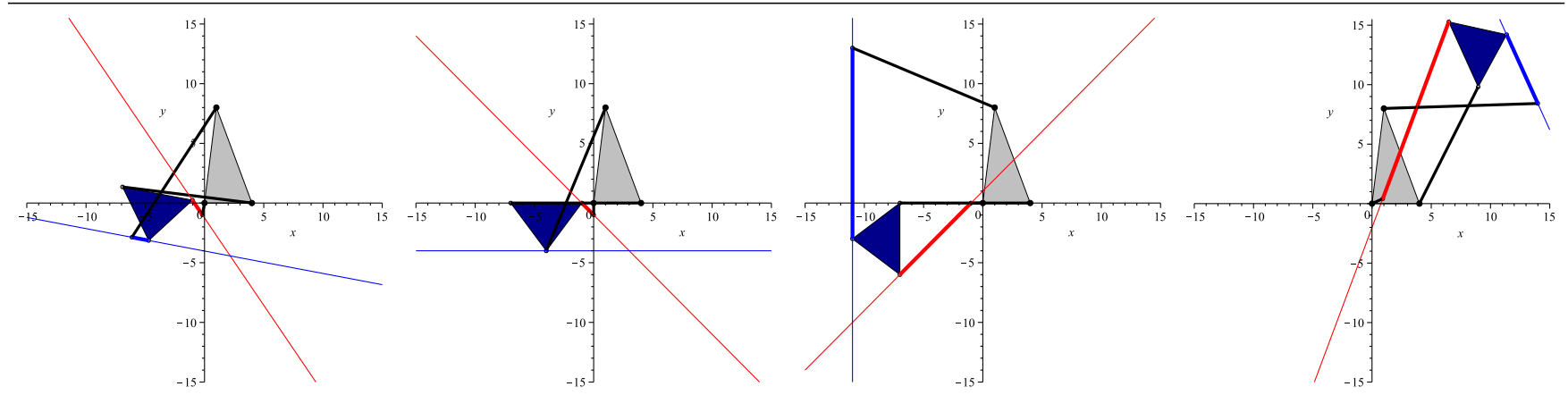

$t=3.9529$

$t=4.0000$

$t=11.0000$

$t=16.2235$

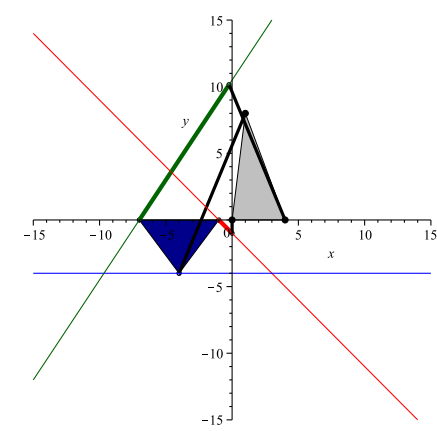

$t=4.0000$

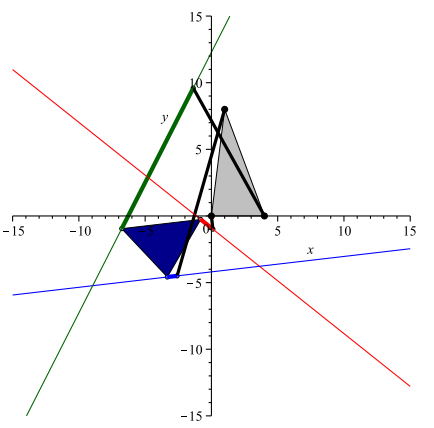

$t=4.1681$

Fig. 5. The solutions of the forward kinematics of the analyzed fully-parallel planar robots (see the Maple Worksheet in the supplementary downloadable multimedia material for the numerical computations). 
sulting polynomial in $\delta_{2}$, we get the univariate polynomial

$$
t^{4}-35.1765 t^{3}+410.7778 t^{2}-1849.7255 t+2821.7516,
$$

where $t$ is the oriented distance between $P_{1}$ and the axis of the prismatic joint that substitutes the revolute joint centered at $P_{6}$.

The real roots of this polynomial are $3.9529,4.0000,11.0000$, and 16.2235. The corresponding solutions of the forward kinematics appear in the third row of Fig. 5

Finally, if the revolute joint centered at $P_{5}$ is also replaced with a prismatic joint, a 3-RPP planar robot platform is obtained. This kind of robot platform belongs to the type VIII robot family in Table III If the orientation of this prismatic joint with respect to its adjacent links, according to the notation of Table III is given by

$$
\begin{aligned}
& \alpha_{7}=2 \pi-\arctan \frac{3}{2}, \\
& \alpha_{8}=2 \pi-\arctan \frac{3}{2}-\arctan \frac{4}{3}, \\
& \alpha_{9}=\pi+\arcsin \frac{3}{\sqrt{13}},
\end{aligned}
$$

then

$$
\begin{aligned}
d_{1,4} & =\delta_{1}+\frac{1}{\sqrt{2}} \\
d_{3,6} & =\delta_{2}-12 \\
d_{1,6} & =\delta_{2}-t \\
d_{4,5}^{2} & =\delta_{1}^{2}+\delta_{3}^{2}+\sqrt{\frac{2}{13}} \delta_{1} \delta_{3}-6 \sqrt{2} \delta_{1}-\frac{6}{\sqrt{13}} \delta_{3}+18 \\
d_{5,6}^{2} & =\delta_{2}^{2}+\delta_{3}^{2}+\frac{4}{\sqrt{13}} \delta_{2} \delta_{3}-8 \delta_{2}-\frac{16}{\sqrt{13}} \delta_{3}+16 \\
d_{4,6}^{2} & =\delta_{1}^{2}+\delta_{2}^{2}+2 \delta_{3}^{2}+\sqrt{\frac{2}{13}} \delta_{1} \delta_{3}+\frac{4}{\sqrt{13}} \delta_{2} \delta_{3} \\
& -6 \sqrt{2} \delta_{1}-8 \delta_{2}-\frac{22}{\sqrt{13}} \delta_{3}+34+\sqrt{2} d_{4,5} d_{5,6}, \\
d_{2,5} & =\delta_{3}-\frac{33}{\sqrt{13}} .
\end{aligned}
$$

Substituting these values in $\Gamma\left(s_{1,6}\right)$, while keeping all other distances unaltered, and iteratively computing the leading coefficients of the resulting polynomial in $\delta_{1}$, and then in $\delta_{2}$, and finally in $\delta_{3}$, we get the univariate polynomial

$$
t^{2}-8.1681 t+16.6723
$$

The real roots of this polynomial are 4.000 and 4.1681 . The corresponding solutions of the forward kinematics appear in the last row of Fig. 5

\section{CONCLUSION}

Regarding a translational motion as an infinitely small rotation about a point at infinity has been a common device to analyze some simple kinematics problems. Applying it to the position analysis of multiloop linkages did not seem to provide any advantage with respect to existing approaches. Nevertheless, it has been shown that, when this idea is combined with a formulation based on distances and oriented areas, the result is a powerful tool that allowed us to conclude that the univariate polynomial closure condition of the 3RPR robot contains the necessary and sufficient information to solve the position analysis of all fully-parallel robots. No new sets of variable eliminations are required.

Moreover, it is worth reminding that the singularities of a fullyparallel planar robot can be obtained by computing the discriminant of its univariate polynomial. Then, according to the presented results, these singularities could be obtained from those of the 3-RPR robot through a limit process. In this sense, it can be said that the presented ideas have far-ranging implications as they can be applied to solve other problems than those tackled in this article.

\section{REFERENCES}

[1] E. Ceresole, P. Fanghella, and C. Galletti, "Assur's groups, AKCS, basic trusses, SOCS, etc.: modular kinematics of planar linkages," Proceedings of the ASME 1996 International Design Engineering Technical Conferences and Computers in Engineering Conference, 96-DETC/MECH-1027, 1996.

[2] S. Li and G. Matthew, "Closed form kinematic analysis of planar Assur II groups," Proceedings of the 7th IFToMM World Congress on the Theory of Machines and Mechanisms, Vol. I, pp. 141-145, 1987.

[3] J. Merlet, "Direct kinematics of planar parallel manipulators," Proceedings of the IEEE International Conference on Robotics and Automation, Vol. 4, pp. 3744-3749, 1996.

[4] S. Mitsi, K.-D. Bouzakis, G. Mansour, and I. Popescu, "Position analysis in polynomial form of planar mechanisms with Assur groups of class 3 including revolute and prismatic joints," Mechanism and Machine Theory, Vol. 38, No. 12, pp. 1325-1344, 2003.

[5] S. Mitsi, K.-D. Bouzakis, and G. Mansour, "Position analysis in polynomial form of planar mechanism with an Assur group of class 4 including one prismatic joint," Mechanism and Machine Theory, Vol. 39, No. 3, pp. 237-245, 2004.

[6] W.-Y. Chung, "The position analysis of Assur kinematic chain with five links," Mechanism and Machine Theory, Vol. 40, No. 9, pp. 1015-1029, 2005.

[7] S. Mitsi, K.-D. Bouzakis, G. Mansour, and I. Popescu, "Position analysis in polynomial form of class-three Assur groups with two or three prismatic joints," Mechanism and Machine Theory, Vol. 43, No. 11, pp. 1401-1415, 2008

[8] M.L. Husty. "Kinematic mapping of planar three-legged platforms," Proceedings of the 15th Canadian Congress of Applied Mechanics (CANCAM 1995), Victoria, B.C., Canada, Vol. 2, pp. 876-877, 1995.

[9] M.J.D. Hayes and P.J. Zsombor-Murray, "A planar parallel manipulator with holonomic higher pairs: inverse kinematics," Proceedings of the CSME Forum 1996, Hamilton, On., Canada, pp. 109-116, 1996.

[10] M.J.D. Hayes, M.L. Husty, and P.J. Zsombor-Murray. "Kinematic mapping of planar Stewart-Gough platforms," Proceedings of the 17th Canadian Congress of Applied Mechanics (CANCAM 1999), Hamilton, On., Canada, pp. 319-320, 1999.

[11] M.J.D. Hayes, Kinematics of General Planar Stewart-Gough Platforms, $\mathrm{PhD}$ thesis, Dept. of Mech. Eng., McGill University, Montréal, Qc., Canada, 1999.

[12] M.J.D. Hayes, M.L. Husty, and P.J. Zsombor-Murray, "Solving the forward kinematics of a planar 3-legged platform with holonomic higher pairs," ASME Journal of Mechanical Design, Vol. 121, No. 2, pp. 212219, 1999.

[13] C. Chen, A Direct Kinematic Computation Algorithm for All Planar 3-Legged Platforms, Master's thesis, Dept. of Mech. Eng., McGill University, Montréal, Qc., Canada, 2001.

[14] P.J. Zsombor-Murray, C. Chen, and M.J.D. Hayes, "Direct kinematic mapping for general planar parallel manipulators," Proceedings of the CSME Forum 2002, Kingston, On., Canada, 2002.

[15] M.J.D. Hayes and M.L. Husty, "On the kinematic constraint surfaces of general three-legged planar robot platforms," Mechanism and Machine Theory, Vol. 38, No. 5, pp. 379-394, 2003.

[16] M.J.D. Hayes, P.J. Zsombor-Murray, and C. Chen, "Unified kinematic analysis of general planar parallel manipulators," Journal of Mechanical Design, Vol. 126, No. 5, pp. 866-874, 2004.

[17] C. Chen and P.J. Zsombor-Murray, Direct Kinematics for All Planar Three-Legged Parallel Platforms, VDM Verlag Publishing House, Saarbrücken, Germany, 2009.

[18] N. Rojas and F. Thomas, "The forward kinematics of 3-RPR planar robots: A review and a distance-based formulation," IEEE Transactions on Robotics, Vol. 27, No. 1, pp 143-150, 2011.

[19] C. Gosselin, J. Sefrioui, and M. Richard, "Solutions polynomiales au problème de la cinématique des manipulateurs parallèles plans à trois degrés de liberté," Mechanism and Machine Theory, Vol. 27, No. 2, pp. 107-119, 1992.

[20] X. Kong and C. Gosselin, "Forward displacement analysis of third-class analytic 3-RPR planar parallel manipulators," Mechanism and Machine Theory, Vol. 36, No. 9, pp. 1009-1018, 2001. 